\title{
Hidden correlations and the behavior of the dynamic structure at short times ${ }^{\text {a) }}$
}

\author{
George D. J. Phillies \\ Department of Chemistry, The University of Michigan, Ann Arbor, Michigan 48109
}

(Received 8 February 1984; accepted 15 March 1984)

\begin{abstract}
Reexamination of methods for calculating the initial slope of the dynamic structure factor demonstrates the importance of covert correlations between the motions and subsequent positions of interacting colloidal particles. These correlations, whose existence has hitherto not been explicitly emphasized, explain the numerical discrepancies between one- and two-time derivative methods for calculating $d S(k, t) / d t$. A method for avoiding artifacts due to these correlations is presented.
\end{abstract}

\section{INTRODUCTION}

The dynamics of suspensions of interacting colloidal particles forms a major topic of current research. ${ }^{1-8}$ Recent successes in the experimental study of concentrated uncharged colloid suspensions, ${ }^{1-3}$ and the extension of Oseentype hydrodynamic interaction tensors to cover three and four particle forces, ${ }^{4}$ suggest that rigorous tests of our understanding of macroparticle dynamics should now be possible. However, before such tests can be made, unambiguous correspondence must be established between experimental data and calculable quantities. In this note, expressions for the mutual diffusion coefficient $D_{m}$ are considered.

Experimentally, $D_{m}$ may be obtained from the dynamic structure factor (intermediate scattering function) $S(k, t)$ by examining its initial slope; by definition

$$
-D_{m} k^{2} S(k, 0)=\lim _{t \rightarrow 0} \frac{d S(k, t)}{d t}
$$

where $k$ is the magnitude of the scattering vector $\mathbf{k} . S(k, t)$ can be determined with quasielastic light scattering spectroscopy and a digital correlator. Since it is physically impossible to measure $S(k, 0)$ with correlation techniques, the limit of Eq. (1) necessarily represents an extrapolation towards $t=0$ from some relatively large time $\sim 50 \mathrm{~ns}-1 \mu \mathrm{s}$.

$S(k, t)$ is related to the instantaneous positions $r_{i}(t)$ of the $N$ scattering particles by

$$
S(\mathbf{k}, t)=N^{-1}\left(\sum_{i, j=1}^{N} \exp \left\{i \mathbf{k} \cdot\left[\mathbf{r}_{i}(0)-\mathbf{r}_{j}(t)\right]\right\}\right),
$$

the brackets denoting an ensemble average. A variety of expressions for the initial slope of $S(k, t)$ exist. For example, Beenacker and Mazur ${ }^{5}$ use

$$
D_{m}=\left\langle N^{-1} \sum_{i, j=1}^{N} \mathrm{D}_{i j} / k_{B} T-\sum_{j=1}^{N} \mathbf{S}_{j}\right\rangle \mathcal{c}\left(\frac{\partial \mu}{\partial c}\right)_{P, T},
$$

where $\mathrm{D}_{i j}$ is the diffusion tensor, $k_{B}$ is Boltzmann's constant, and $S_{j}$ is the fluid mobility - the flow of fluid induced by the motion of particle $j$. Equation (3) appears nearly identical to the form obtained ${ }^{6}$ by Akcasu and Gurol for the diffusion of beads of a polymer chain. If one neglects hydrodynamic interactions, Eq. (3) reduces to the expression

$$
\begin{aligned}
\frac{d S(k, t)}{d t}= & -N^{-1}\left\langle\sum_{i, j=1}^{N} k^{2} D_{0} \delta_{i j}\right. \\
& \left.\times \exp \left\{l \mathbf{k} \cdot\left[\mathbf{r}_{i}(0)-\mathbf{r}_{j}(0)\right]\right\}\right\rangle,
\end{aligned}
$$

a) The support of this work by the National Science Foundation under Grant CHE82-13941 is gratefully acknowledged. of Ackerson et al., ${ }^{7}$ where $D_{0}=\operatorname{tr}(\mathrm{D}) / 3$. On the other hand, this author ${ }^{8}$ obtained the form

$$
\begin{aligned}
\frac{d S(k, t)}{d t}= & N^{-1}\left\{\sum_{i, j=1}^{N} \exp \left\{i \mathbf{k} \cdot\left[\mathbf{r}_{i}(0)-\mathbf{r}_{j}(0)\right]\right\}\right. \\
& \left.\times\left[-i \mathbf{k} \cdot \mathbf{v}_{j}(t)-\mathbf{k} \cdot \mathrm{D}_{i j} \cdot \mathbf{k}-\mathbf{k} \cdot \Delta \mathrm{D} \cdot \mathbf{k}\right]\right\rangle,
\end{aligned}
$$

where $\mathbf{v}_{j}(t)$ is the velocity of particle $j$ at time $t(t \neq 0)$, and where $\Delta \mathrm{D}$ is the dynamic friction term due to correlations between the Brownian displacement and the subsequent interparticle forces on each particle.

Equations (4) and (5) are obtained by the seemingly innocuous application of elementary calculus, followed by use of Kubo relations, such as

$$
\left\langle\int_{0}^{t} d s \mathbf{v}_{B i}(0) \mathbf{v}_{B i}(s)\right\rangle=\mathrm{D}_{i i},
$$

between the diffusion coefficients and the Brownian velocity $\mathbf{v}_{B i}$ of a particle. Unfortunately, Eqs. (4) and (5) are numerically inequivalent. On rearrangement, Eq. (5) is found to contain nonzero terms in $\nabla \cdot\left(D_{i j}\right)$, as well as the nonzero $\Delta D$ term, while Eq. (4) lacks such terms. The differences between these equations have largely been ignored, or ascribed to the neglect of unspecified hidden correlations in the particle motions. The objective of this note is to locate the hypothesized hidden correlations, thereby demonstrating which of Eqs. (3) $-(5)$ is to be preferred.

Section II of this paper proceeds as far as possible towards Eqs. (3)-(5) without using model-dependent assumptions. In Sec. III, a model for particle motion based on the generalized Langevin equation is introduced. Equations equivalent to Eqs. (4) and (5) are obtained in Sec. IV; these equations are shown to have equally valid derivations, but not to be equivalent. In Sec. V, correlations hidden in the Langevin equation are revealed; it is argued that by structuring the calculation correctly the hidden correlations can be avoided. A discussion is found in Sec. VI.

\section{MODEL-INDEPENDENT RESULTS}

$S(k, t)$ only depends on the time interval between the two moments [in Eq. (2) 0 and $t$ ] at which the particle positions are known. Denoting these two moments by $\tau$ and $T$, with $t=T-\tau$, and invoking the physical requirement that particle positions are continuous functions of the time, one has

$$
\begin{aligned}
\frac{d S(k, t)}{d t}= & N^{-1}\left\langle\sum_{i, j=1}^{N}-i \mathbf{k} \cdot \mathbf{v}_{j}(T)\right. \\
& \left.\times \exp i \mathbf{k} \cdot\left[\mathbf{r}_{i}(\tau)-\mathbf{r}_{j}(T)\right]\right\rangle,
\end{aligned}
$$


$v_{j}$ being a particle velocity.

To use equilibrium statistical mechanics to evaluate Eq. (7), the particle positions must be referred to the same time, as is done by introducing the displacement

$$
\delta \mathbf{r}_{i}=\int_{\tau}^{T} d u \mathbf{v}_{i}(u)
$$

and expanding the exponential as

$$
e^{i \mathbf{k} \cdot[\mathbf{r}+\delta \mathbf{r}]}=e^{\mathbf{k} \cdot \mathbf{r}}[1+i \mathbf{k} \cdot \delta \mathbf{r}+\cdots] .
$$

To obtain $d S / d t$ to order $k^{2}$, only the lead terms of the expansion are required. In Eq. (7), $\mathbf{r}_{j}$ may be expanded around the moment $\tau$, or $\mathbf{r}_{i}$ may be expanded around the moment $T$, so

$$
\begin{aligned}
\left(\frac{d S}{d t}\right)_{a}= & N^{-1}\left\langle\sum_{i, j=1}^{N} \exp \left\{\mathbf{i k} \cdot\left[\mathbf{r}_{i}(\tau)-\mathbf{r}_{j}(\tau)\right]\right\}\right. \\
& \left.\times\left[-i \mathbf{k} \cdot \mathbf{v}_{j}(T)-\int_{\tau}^{T} d u \mathbf{k} \cdot \mathbf{v}_{j}(u) \mathbf{k} \cdot \mathbf{v}_{j}(T)\right]\right),
\end{aligned}
$$

or

$$
\begin{aligned}
\left(\frac{d S}{d t}\right)_{b}= & N^{-1}\left(\sum_{i, j=1}^{N} \exp i \mathbf{k} \cdot\left[\mathbf{r}_{i}(T)-\mathbf{r}_{j}(T)\right]\right. \\
& \left.\times\left[-i \mathbf{k} \cdot \mathbf{v}_{j}(T)-\int_{\tau}^{T} d u \mathbf{k} \cdot \mathbf{v}_{i}(u) \mathbf{k} \cdot \mathbf{v}_{j}(T)\right]\right),
\end{aligned}
$$

where Eq. (8) has been applied to write the displacement $\delta r$ in terms of an integral over the particle velocity. As will be seen below, the Eqs. (3)-(5) for $D_{m}$ may be obtained from $(\partial S / \partial t)_{a}$ or $(\partial S / \partial t)_{b}$.

The above computation used only the techniques of freshman calculus; differentiation, integration, and expansion in power series to a specified order in $k$. No arguments based on time scales, models for particle motion, or stationarity were invoked. As long as the power series are convergent, $(d S / d t)_{a}$ and $(d S / d t)_{b}$ must be equal. However, these derivations are not identical:

(i) In Eq. (10a) the fixed time $T$, at which $\mathbf{v}_{j}$ is specified, is different than the time $\tau$ at which the particle positions are specified, while in Eq. $(10 \mathrm{~b})$ the fixed time $T$, at which $\mathbf{v}_{j}$ is specified, is the same as the time at which the particle positions are specified.

(ii) In Eq. (10a), the velocities in the correlation integral $\int \mathbf{v}_{j}(u) \mathbf{v}_{j}(T)$ are those of the same particle $j$ at two times, while in Eq. (10b) the velocities in the correlation integral $\int \mathbf{v}_{i}(u) \mathbf{v}_{j}(T)$ are those of two different particles at two times.

\section{MODEL FOR PARTICLE MOTION}

The model for particle motions used here is the generalized Langevin equation

$$
M \frac{d \mathbf{v}_{i}}{d t}=-f \cdot v_{i}+F_{B i}-\nabla_{i} W,
$$

in its low-frequency form

$$
M \frac{d \mathbf{v}_{i}}{d t} \cong 0,
$$

where $M$ is the macroparticle mass, $f$ is the Stokes' Law drag coefficient, $\mathbf{F}_{B i}$ is the fluctuating force on the macroparticle due to random solvent motions, and $-\nabla W$ is the force on $i$ due to the intermacroparticle potential $W$. At long times the
Langevin equation is equivalent to the Smoluchowski equation. ${ }^{9}$ A particle has a drift velocity

$$
\nabla_{D i}=-\nabla W \cdot f^{-1}
$$

and a fluctuating Brownian velocity component

$$
\mathbf{v}_{B i}=\mathbf{v}_{i}-\mathbf{v}_{D i} \text {. }
$$

The drift velocity of Eq. (13) is the same as the drift velocity found in the Smoluchowski sedimentation equation. In the absence of external forces, for identical particles, $\mathbf{v}_{D i}$ has the significant property

$$
\sum_{i=1}^{N} \mathbf{v}_{D i}=0
$$

which follows from the Newton's law requirement that forces (and hence drift velocities) of a pair of interacting particles are equal and opposite.

Stokes' Law is a low frequency approximation. If one applies a force on a particle, there is some short time $\tau_{H}$ before the surrounding solvent molecules reach their steady state behavior. As shown in Rice and Gray, ${ }^{10}$ this time is shorter than the correlation time $\tau_{B}$ of Eq. (16). The Brownian velocity component is usually assumed to have a very short correlation time $\tau_{B}$ :

$$
\left\langle\mathbf{v}_{B i}(0) \mathbf{v}_{B i}(t)\right\rangle=0, \quad t>\tau_{B} .
$$

The random motions are also generally assumed to be decoupled from the slowly varying drift velocity, ${ }^{9}$ so that the Kubo relation applies

$$
\left\langle\int_{0}^{t} \mathbf{v}_{B i}(0) \mathbf{v}_{B i}(u) d u\right\rangle=\mathrm{D}_{i i}, \quad t>\tau_{B} .
$$

Hydrodynamic interactions between the Brownian macroparticles lead to well-known ${ }^{4,11,12}$ modifications in Eqs. (13) and (17).

The energy of a system of macroparticles may be decomposed into kinetic energy terms dependent only on particle velocities and potential energy terms dependent only on particle positions. In the canonical ensemble, at a given instant in time the particle positions and velocities are therefore independent, so that

$$
\begin{aligned}
\left\langle\exp \left\{\boldsymbol{i} \mathbf{k} \cdot\left[\mathbf{r}_{i}(T)-\mathbf{r}_{j}(T)\right]\right\} i \mathbf{k} \cdot \mathbf{v}_{j}(T)\right\rangle \\
\quad=\left\langle\exp \left\{i \mathbf{k} \cdot\left[\mathbf{r}_{i}(T)-\mathbf{r}_{i}(T)\right]\right\}\right\rangle\left\langle i \mathbf{k} \cdot \mathbf{v}_{j}(T)\right\rangle=0
\end{aligned}
$$

the final equality following from the reflection symmetry of the kinetic energy.

\section{REDUCTION OF $d S(k, t) / d t$}

Equations (11)-(18) are now used to reduce Eqs. (10a) and (10b) to simpler form. From Eq. (18), the part of Eq. (10b) which is linear in $\mathbf{v}$ vanishes. Equation (15) remains true if each term is multiplied by the same constant, so to order $k^{2}$ :

$$
\begin{aligned}
\sum_{j=1}^{N}[ & \exp i \mathbf{k} \cdot\left[\mathbf{r}_{i}(T)-\mathbf{r}_{j}(T)\right] \\
& \left.\times \int d u \mathbf{k} \cdot \mathbf{v}_{i}(u)\right] \mathbf{k} \cdot \mathbf{v}_{D_{j}}(T)=0 .
\end{aligned}
$$

If one substitutes $v_{i}=v_{B i}+v_{D i}$ and uses Eq. (19), Eq. (10b) reduces to 


$$
\begin{aligned}
\left(\frac{d S}{d t}\right)_{b}= & -\lim _{t \rightarrow 0} N^{-1}\left(\sum_{i, j=1}^{N} \exp \left\{i \mathbf{k} \cdot\left[\mathbf{r}_{i}(T)-\mathbf{r}_{j}(T)\right]\right\}\right. \\
& \left.\times \int_{\tau}^{T} d u \mathbf{k} \cdot \mathbf{v}_{B i}(u) \mathbf{k} \cdot \mathbf{v}_{B j}(T)\right)
\end{aligned}
$$

In Eq. (10a), the linear term in

$$
\left\langle\exp \left[\boldsymbol{i} \mathbf{k} \cdot\left(\mathbf{r}_{i}(\tau)-\mathbf{r}_{j}(\tau)\right)\right] \mathbf{k} \cdot \mathbf{v}_{B j}(T)\right\rangle
$$

vanishes, because between $\tau$ and $T$ the Brownian velocity will have thermalized, losing any possible correlation with $\mathbf{r}_{i}(\tau)$. Equation (10a) becomes

$$
\begin{aligned}
\left(\frac{d S}{d t}\right)_{a}= & \lim _{t \rightarrow 0} N^{-1}\left(\sum_{i, j=1}^{N} \exp \left\{i \mathbf{k} \cdot\left[\mathbf{r}_{i}(\tau)-\mathbf{r}_{j}(\tau)\right]\right\}\right. \\
& \times\left[-i \mathbf{k} \cdot \mathbf{v}_{D j}(T)-\int_{\tau}^{T} d u \mathbf{k} \cdot \mathbf{v}_{B j}(u) \mathbf{k} \cdot \mathbf{v}_{B j}(T)\right. \\
& -\int_{\tau}^{T} d u \mathbf{k} \cdot \mathbf{v}_{B j}(u) \mathbf{k} \cdot \mathbf{v}_{D j}(T) \\
& \left.\left.-\int_{\tau}^{T} d u \mathbf{k} \cdot \mathbf{v}_{D j}(u) \mathbf{k} \cdot \mathbf{v}_{B j}(t)\right]\right) .
\end{aligned}
$$

If $T-\tau$ is small, $v_{D i}(u)$ is nearly constant over the interval $(T, \tau) ;$ the omitted term in

$$
\int d \mu \mathbf{v}_{D i}(u) \mathbf{v}_{D i}(T)
$$

is linear in $t$, and vanishes as $t \rightarrow 0$. By comparison with Eq. (5), we define

$$
\begin{aligned}
\mathbf{k} \cdot \Delta \mathrm{D} \cdot \mathbf{k}= & \int_{\tau}^{T} d u \mathbf{k} \cdot \mathbf{v}_{B j}(u) \mathbf{k} \cdot \mathbf{v}_{D j}(T) \\
& +\int_{\tau}^{T} d u \mathbf{k} \cdot \mathbf{v}_{D j}(u) \mathbf{k} \cdot \mathbf{v}_{B j}(T) .
\end{aligned}
$$

Finally, from the Kubo relation (17), one generally infers

$$
\begin{gathered}
\left\langle\sum_{i, j=1}^{N} \exp \tau\left\{\boldsymbol{l} \mathbf{k} \cdot\left[\mathbf{r}_{i}(\tau)-\mathbf{r}_{j}(\tau)\right]\right\} \int_{\tau}^{T} d u \mathbf{k} \cdot \mathbf{v}_{B j}(u) \mathbf{k} \cdot \mathbf{v}_{B j}(T)\right\rangle \\
=\left\langle\sum_{i, j=1}^{N} e^{i \mathbf{k} \cdot\left[\mathbf{r}_{i}-\mathbf{r}_{j}\right]} \mathbf{k} \cdot \mathrm{D}_{j j} \cdot \mathbf{k}\right\rangle
\end{gathered}
$$

and

$$
\begin{gathered}
\left\langle\sum_{i, j=1}^{N} \exp \left\{\boldsymbol{i k} \cdot\left[\mathbf{r}_{i}(T)-\mathbf{r}_{j}(T)\right]\right\} \int_{\tau}^{T} d u \mathbf{k} \cdot \mathbf{v}_{B i}(u) \mathbf{k} \cdot \mathbf{v}_{B j}(T)\right\rangle \\
=\left\langle\sum_{i, j=1}^{N} e^{i \mathbf{k} \cdot\left[\mathbf{r}_{i}-\mathbf{r}_{j}\right]} \mathbf{k} \cdot \mathrm{D}_{i j} \cdot \mathbf{k}\right\rangle
\end{gathered}
$$

Here we have used the argument that $\exp \left[i \mathbf{k} \cdot\left(\mathbf{r}_{i}-\mathbf{r}_{j}\right)\right]$ is essentially a constant over the time $\tau_{B}$ during which $\int d u \mathbf{v}_{B}(u) \mathbf{v}_{B}(T)$ is nonzero, so that the average over $\mathbf{v}_{B}$ is not affected by differences between the $r_{i}(\tau)$ of Eq. (23a) and the $r_{i}(T)$ of Eq. (23b).

The above considerations serve to reduce Eq. (10) to a more familiar form. As shown in Appendix A, if one expands $\mathrm{D}_{i j}$ as

$$
\begin{aligned}
\mathrm{D}_{i j}= & D_{0} \delta_{i j}+k_{B} T\left(\sum_{l} \mathrm{~b}_{i l}+\sum_{l, m} \mathrm{~b}_{i l m}\right) \delta_{i j} \\
& +k_{B} T\left(\mathrm{~T}_{i j}+\sum_{m} \mathrm{~T}_{i m j}\right)\left(1-\delta_{i j}\right),
\end{aligned}
$$

where $T_{i j}$ and $T_{i m j}$ are the two and three particle Oseen ten- sors, and $b_{i l}$ and $b_{i I m}$ represent the effect on the mobility of $i$ of reflections by $l$ and $m$ of the hydrodynamic wake of $i$, one obtains

$$
\begin{aligned}
\left(\frac{d S}{d t}\right)_{a}= & N^{-1}\left\langle-\sum_{i, j} e^{i \mathbf{k} \cdot \mathbf{r}_{i j}}\left(\mathbf{k} \cdot \mathrm{D}_{i j} \cdot \mathbf{k}\right)\right\rangle \\
& +\left\langle\sum_{i j l} e^{i \mathbf{k} \cdot \mathbf{r}_{i j}}\left(i \mathbf{k} \nabla_{l}: \mathrm{D}_{j l}\right)\right\rangle-\mathbf{k} \cdot \Delta \mathrm{D} \cdot \mathbf{k},
\end{aligned}
$$

but

$$
\left(\frac{d S}{d t}\right)_{b}=N^{-1}\left(-\sum_{i, j} e^{i \mathbf{k} \cdot \mathbf{r}_{i j}}\left(\mathbf{k} \cdot \mathrm{D}_{i j} \cdot \mathbf{k}\right)\right\rangle
$$

Equation (25a) is equivalent to Eq. (5), while with the proper hydrodynamic tensors Eq. (25b) takes the form of Eq. (3) or Eq. (4), confirming the above assertion that Eqs. $(3)-(5)$ all follow from Eq. (10a) or Eq. (10b).

One of the major results of the modern hydrodynamic theory of interacting spheres, emphasized by Felderhof, ${ }^{11}$ is

$$
\nabla_{l} \cdot \mathrm{D}_{j l} \neq 0
$$

if $\mathbf{D}_{j l}$ is expanded to high order in $(a / r)$. Here $a$ is the radius of a sphere, while $r$ is the distance between the spheres. While an incompressible fluid satisfies $\nabla \cdot v=0$ for its own flow, the Faxen theorem insures that the velocity of a sphere in a nonuniform flow is not the same as the velocity which the fiuid would have had, at the sphere's center, if the sphere had been absent. The motion of the spheres, relative to a divergenceless fluid flow, need not be divergenceless.

If ik $\nabla_{l}: \mathrm{D}_{j l}$ does not vanish, Eqs. (25a) and (25b) do not agree, even though these results were obtained by applying a single physical model to algebraically equivalent starting points. The discrepancy does not go away if one neglects hydrodynamic interactions, i.e., if $D_{i j}=D_{0} \delta_{i j}$. As shown by Ackerson et al., ${ }^{7}$ for $T-\tau$ small

$$
\begin{aligned}
& N^{-1}\left(\sum_{i, j=1}^{N} \exp \left\{\mathbf{i k} \cdot\left[\mathbf{r}_{i}(\tau)-\mathbf{r}_{j}(\tau)\right]\right\}\left[-\boldsymbol{i k} \cdot \mathbf{v}_{D j}(T)\right]\right) \\
& =\frac{k_{B} T}{f} k^{2}[S(k)-1] .
\end{aligned}
$$

In this case, Eqs. (25) becomes

$\left(\frac{d S}{d t}\right)_{a}=D_{0} k^{2}[S(k)-1]-D_{0} k^{2} S(k)-\mathbf{k} \cdot \Delta \mathrm{D} \cdot \mathbf{k} S(k)$,

$\left(\frac{d S}{d t}\right)_{b}=-D_{0} k^{2}$

An explicit calculation, ${ }^{8}$ now qualitatively supported by experiment, ${ }^{13}$ shows that $\Delta D \neq 0$. Even if $D_{i j}$ does not incorporate interparticle interactions, Eqs. (28) indicate $(d S / d t)_{a}$ $\neq(d S / d t)_{b}$.

\section{RESOLUTION OF THE ANOMALY IN $(d S / d t)$}

The manipulations leading to Eqs. (10a) and (10b) are unexceptionable, so the inequivalence of $(d S / d t)_{a}$ and $(d S / d t)_{b}$ is most reasonably attributed to some subtle feature of the model for particle motion given in Sec. III. That is, Eqs. (25a) and (25b) are presumably unequal because correlations between $v_{B}, v_{D}$, and the particle positions have been neglected. This section shows where and when these 
correlations are hidden and argues how artifacts due to these correlations may be avoided.

The correlations will be revealed by considering precisely what one means by the drift velocity. This author ${ }^{8}$ and Pusey and Tough ${ }^{14}$ give differing physical interpretations of Eqs. (13) and (14), but the hidden correlations are predicted to occur at the same point. The resolution of the problem will be to arrange the computations to avoid the correlations; agreement on a more precise physical interpretation of the drift velocity will not be needed.

Pusey and Tough argue that $\mathbf{v}_{D}$ is only defined on time scales longer than $\tau_{H}$, the time scale on which the frequencyindependent Stokes' Law is valid. If the particle positions are specified at a time $t, \mathbf{v}_{D}(t)$ is zero and $\mathbf{v}(t)=\mathrm{v}_{B}(t)$. Contrarily, this author has proposed that since $W$ is slowly varying, and since $W$ acted prior to the moment $t$, Eq. (13) defines $v_{D}$, while $\nabla_{B}$ is simply the difference between $\mathbf{v}_{D}$ and $\mathbf{v}$.

For a freely moving particle, $v_{B}$ is commonly envisioned as being driven by random stress fluctuations in the solvent. If one neglects interparticle hydrodynamics, the Brownian motions of neighboring particles are not correlated with the particle positions. These images, which appear in the interacting particle model as Eqs. (17) and (23), are not quite consistent with either interpretation of $\mathbf{v}_{D}$. At moments other than $t, v_{B}$ may be said to be due to solvent fluctuations. However, in the interpretation of Pusey and Tough, $\mathbf{v}_{D}(t)$ vanishes. As interparticle interactions still act at $t$, at the unique moment $t \mathbf{v}_{B}$ includes a contribution to particle motion due to interparticle interactions. $\mathbf{v}_{B}(t)$ and $\mathbf{v}_{B}(T)$ [where $T-t>\tau_{B}$ ] thus do not have the same physical causes. At the moment $t$, it is then not physically transparent that $\int d u v_{B}(u) v_{B}(t)$ is given by the free particle value of $\mathrm{Eq}$. (17), since $v_{B}$ is generally driven only by solvent stress fluctuations, while $v_{B}(t)$ is also driven by the interparticle interactions.

On the other hand, if one defines $\mathbf{v}_{D}$ by Eq. (13), then $\mathbf{v}_{B}(t)$ and the $\mathbf{r}_{i}(t)$ are correlated. Namely, from Eq. (18),

$$
\begin{aligned}
0= & \left\langle\exp \left\{\boldsymbol{i k} \cdot\left[\mathbf{r}_{i}(t)-\mathbf{r}_{j}(t)\right]\right\} i \mathbf{k} \cdot \mathbf{v}_{j}(t)\right\rangle \\
= & \left\langle\exp \left\{\boldsymbol{i k} \cdot\left[\mathbf{r}_{i}(t)-\mathbf{r}_{j}(t)\right]\right\} i \mathbf{k} \cdot \mathbf{v}_{B j}(t),\right. \\
& +\left\langle\exp \left\{i \mathbf{k} \cdot\left[\mathbf{r}_{i}(t)-\mathbf{r}_{j}(t)\right]\right\} i \mathbf{k} \cdot \mathbf{v}_{D j}(t)\right\rangle
\end{aligned}
$$

or

$$
\begin{aligned}
& \left\langle\exp \left\{\boldsymbol{i} \mathbf{k} \cdot\left[\mathbf{r}_{i}(t)-\mathbf{r}_{j}(t)\right]\right\} i \mathbf{k} \cdot \mathbf{v}_{B j}(t)\right\rangle \\
& \quad=\left\langle\exp \left\{i \mathbf{k} \cdot\left[\mathbf{r}_{i}(t)-\mathbf{r}_{j}(t)\right]\right\} i \mathbf{k} \cdot \nabla_{j} W\left(r_{i}, r_{j}\right) \cdot f^{-1}\right\rangle, \quad \neq 0,
\end{aligned}
$$

which is inconsistent with the argument for Eq. (23).

It should be possible to remove these problems with projection operators or more careful analysis. A simpler approach is to rely on Eq. (16). Positions and velocities taken at the same time may have subtle correlations, but if one waits a period $>\tau_{B}$, initial correlations between $\mathbf{v}_{B}$ and $\mathbf{v}_{D}$ or $\mathbf{r}_{i}$ will decay away. A calculation which specifies particle positions at a time $T$, and uses particle velocities only at time $t$ (with $\left.|T-t|>\tau_{B}\right)$, will not be affected by hidden correlations, because by $t \nabla_{B}$ will be thermalized, and $\nabla_{D}$ will have reached its low-frequency drift value. By inspection, Eq. (10a) satisfies this approach by correlating $\mathbf{r}_{i}(\tau)$ and $\mathbf{v}(T)$, but Eq. (10b) correlates $\mathbf{r}_{j}$ and $v_{j}$ at the same moment $T$. If hidden correla- tions are responsible for the differences between Eqs. (25a) and (25b), the fault is in Eq. (25b), not in Eq. (25a).

\section{DISCUSSION}

In the above, two alternative methods of expanding $d S$ / $d t$ at short times were considered. These amounts give expressions which are algebraically equivalent to results previously obtained with other techniques. ${ }^{8.7}$ While the expansions of Eq. (10) appear algebraically equivalent, in that both of them are based on a power series to a given order in $k$, the expansions give different numerical answers for the mutual diffusion coefficient $D_{m}$. In Sec. V, it was argued that the discrepancy arises from the neglect of subtle correlations between particle motions and positions. These correlations were not evaluated directly. A consideration of the model for particle motions indicates that these correlations are most likely to be handled incorrectly if particle positions and Brownian velocities are averaged at the same moment in time, as opposed to averaging the particle positions at one moment, and the particle Brownian velocities at another moment. Equation (25a) for $d S / d t$ is therefore to be preferred to Eq. (25b), in accord with our previous work. . $^{8.19}$

Historically, ${ }^{9}$ the Langevin equation (and the corresponding Smoluchowski equation) were applied to cases (harmonically bound particle, sedimentation) in which changes in the fluctuating force $F_{B}$ and in the external force $-\nabla_{i} W$ could be presumed to be uncorrelated. As noted by Chandrasekhar, ${ }^{15}$ in obtaining the Langevin and Smoluchowski equations one has made the drastic assumption that the forces can be divided into two uncorrelated ${ }^{16}$ parts. In the cases discussed by Chandrasekhar, the force $\mathbf{F}_{B}$ was assumed to fluctuate several times during an interval so short that $-f v_{i}$ only changed by a small amount. This assumption was clearly physically reasonable. In contrast, in the colloidal diffusion problem considered here, $\mathbf{F}_{B}$ and $\nabla_{i} W$ both fluctuate on molecular time and distance scales. The usual methods for obtaining the Smoluchowski equation from a Fokker-Planck equation ${ }^{17}$ will still be valid, but $\mathbf{F}_{B}$ and $\nabla_{i} W$ can no longer be assumed to be independent. In the presence of these correlations, the Smoluchowski equation form

$$
\lim _{t \rightarrow 0} \frac{d S}{d t}=\left\langle\sum_{i, j=1}^{N}\left\{\nabla_{i} \cdot \mathrm{D}_{s m} \cdot \nabla_{j}-\nabla_{i} \cdot \mathrm{D}_{s m} \cdot \beta \nabla_{j}[W]\right\} e^{i \mathbf{k} \cdot\left(\mathbf{r}_{i}-\mathbf{r}_{j}\right)}\right\rangle
$$

for the time dependence of the dynamic structure factor should be correct, if $\mathrm{D}_{s m}$ is interpreted correctly. Here $\beta=\left(k_{B} T\right)^{-1}$. Equations (25a) and (31) agree if the diffusion coefficient $\mathrm{D}_{s m}$ of the Smoluchowski equation is a dressed diffusion coefficient

$$
\mathrm{D}_{s m}=\mathrm{D}_{i j}+\Delta \mathrm{D}
$$

incorporating the effect of correlations between $F_{B}$ and $\nabla_{i} W$. [Equation (31) has $\boldsymbol{\nabla} \cdot \mathrm{D}$ terms.]

If $D_{m}$ were to be obtained from the Smoluchowski equation (31) with the assumption that $D_{s m}$ is a bare diffusion coefficient $D_{i j}$ or $D_{0} \delta_{i j}$, no $\Delta \mathrm{D}$ term would be found in $D_{m}$, because interpreting $\mathrm{D}_{s m}$ as the bare diffusion coefficient is equivalent to assuming that $\Delta \mathrm{D}=0$. This (negative) prediction is confirmed by extensive calculations of $d S / d t$, using 
the Mori formalism and the Smoluchowski equation with $D_{m}=D_{0}$; such computations find no sign of a $\Delta \mathrm{D}$ term through order $\left(k^{2}\right)$ and first order in time. ${ }^{18}$

The physical nature of the dynamic friction term may be more readily understood by considering an extreme case of intermacromolecular interaction: the formation of a dimer from two monomers. Suppose one has two noninteracting particles, whose Brownian displacements over some period of time are $\mathbf{R}_{1}$ and $\mathbf{R}_{2}$, respectively. A covalent link between the two monomers will serve to distribute the force on each particle over both particles of the dimer. By. Eq. (15), any reduction $\mathbf{\delta R}$ in the displacement of one particle of the dimer is exactly matched by an increase in the displacement of the other particle. To illustrate this effect, consider the special case in which $\mathbf{R}_{1}$ and $\delta \mathbf{R}$ are directed along the line centers of the two particles. In this case, the fluctuating force responsible for $\mathbf{R}_{1}$ will be resisted equally by both particles; instead of moving the first particle through $\mathbf{R}_{1}$, the fluctuating force will move each particle of a linked pair through an equal distance $\mathbf{R}_{1} / 2$.

Superficially, then, the interparticle interaction appears to have no effect on the mass flow $m \Delta x$; instead of moving a monomer of mass $m$ through $\mathbf{R}_{1}$, because of the interaction, twice the mass is displaced by half the distance, so $m \Delta x$ appears the same with or without the interparticle bonding. However, the bond distributes the random forces on both particles evenly over both particles. If $\mathbf{R}_{1}$ and $\mathbf{R}_{2}$ are much larger than the bond length, the effect of the bond is to cause the first particle to be displaced by $\left(\mathbf{R}_{1}+\mathbf{R}_{2}\right) / 2$ rather than $\mathbf{R}_{1}$, and similarly for $\mathbf{R}_{2}$. Uncorrelated random variables add incoherently. The distribution of $\left(\mathbf{R}_{1}+\mathbf{R}_{2}\right) / 2$ is narrower than the distributions of $\mathbf{R}_{1}$ and $\mathbf{R}_{2}$, so the mean-square displacement of a particle in a dimer is less than the meansquare displacement of the same particle when it is free. Physically, the $\Delta \mathrm{D}$ term acts by averaging the random force $\mathrm{F}_{B}$ on each particle in an interacting cluster over all of the particles in a cluster. The average of several random forces has a narrower distribution than a single random force. The averaging diminishes the diffusion coefficient of the individual particles.

\section{APPENDIX: $(d S / d t)_{a}$ and $(d S / d t)_{b}$ IN THE PRESENCE OF HYDRODYNAMIC INTERACTIONS}

The goal of this Appendix is to obtain Eq. (25a). From Eqs. (21), (22), and (23a), one has

$$
\begin{aligned}
\left(\frac{d S}{d t}\right)_{a}= & N^{-1}\left\langle\sum_{i, j=1}^{N} \exp i \mathbf{k} \cdot\left[\mathbf{r}_{i}(\tau)-\mathbf{r}_{j}(\tau)\right]\right\rangle \\
& \left.\times\left[-i \mathbf{k} \cdot \mathbf{v}_{D j}(T)-\mathbf{k} \cdot \mathrm{D}_{j j} \mathbf{k}-\mathbf{k} \cdot \Delta \mathrm{D} \cdot \mathbf{k}\right]\right\rangle .
\end{aligned}
$$

A compressed notation for the mobility tensor is

$$
\begin{aligned}
& \mu_{i i}=\sum_{l \neq i} \mathrm{~b}_{i l}+\sum_{\substack{l, m \\
\neq i}} \mathrm{~b}_{i l m}, \\
& \mu_{i j}=\mathrm{T}_{i j}+\sum_{m \neq i j} \mathrm{~T}_{i m j}, \quad i \neq j,
\end{aligned}
$$

where the leading terms of the $b$ and $T$ tensors are ${ }^{4}$

$$
\mathrm{b}_{i l}=-\frac{15}{4}\left(\frac{a}{r_{i l}}\right)^{4} \hat{r}_{i l} \hat{r}_{i l}
$$

$$
\begin{aligned}
\mathrm{b}_{i m l}= & \frac{75 a^{6}}{16 r_{i m}^{2} r_{i l}^{2} r_{m l}^{3}}\left\{\left[1-3\left(\hat{r}_{i m} \cdot \hat{r}_{m l}\right)^{2}\right]\right. \\
& \times\left[1-3\left(\hat{r}_{m l} \cdot \hat{r}_{i l}\right)^{2}\right]+6\left[\hat{r}_{i m} \cdot \hat{r}_{m l}\right]^{2}\left[\hat{r}_{m l} \cdot \hat{r}_{i l}\right]^{2} \\
& \left.-6\left(\hat{r}_{i m} \cdot \hat{r}_{i l}\right)\left(\hat{r}_{i m} \cdot \hat{r}_{m l}\right)\left(\hat{r}_{i l} \hat{r}_{m l}\right)\right\} \hat{r}_{i m} \hat{r}_{i l}, \\
\mathrm{~T}_{i j}= & \frac{3}{4}\left(\frac{a}{r_{i j}}\right)\left[1+\hat{r}_{i j} \hat{r}_{i j}\right], \\
\mathrm{T}_{i m l}= & -\frac{15}{8} \frac{a^{4}}{r_{i m}^{2} r_{m l}^{2}}\left[1-3\left(\hat{r}_{i m} \cdot \hat{r}_{m l}\right)^{2}\right] \hat{r}_{i m} \hat{r}_{m l},
\end{aligned}
$$

where $a$ is the sphere radius, $r_{i j}$ is the scalar distance between $i$ and $j, \hat{r}_{i j}$ is the unit vector from $i$ to $j$, I is the identity tensor, $\hat{r} \hat{r}$ denotes the outer (dyadic) product, and only the lowest order term (in $a / r$ ) of each tensor has been given.

The diffusion tensor and the drift velocity may be written

$$
\begin{aligned}
& \mathrm{D}_{i j}=D_{0} \delta_{i j}+k_{B} T \mu_{i j}, \\
& \mathbf{v}_{D j}=\mu \cdot \mathbf{F}_{j}+\sum_{l \neq j} \mu_{j l} \cdot \mathbf{F}_{l},
\end{aligned}
$$

where $\delta_{i j}$ is the Kronecker delta and $\mathbf{F}_{l}$ is the force on $l$. Simplification follows closely on the lines of an earlier paper. ${ }^{19}$ If the ensemble average over particle positions is normalized as

$$
\langle\cdots\rangle=\int \frac{d r^{N} e^{-\beta(W-A)}}{V^{N}}(\cdots),
$$

$\int d \mathbf{r}^{N}$ being the integral over the $N$ particle coordinates, $\beta$ being $\left(k_{B} T\right)^{-1}$, and $V$ being the volume of the system, Eq. (A1) becomes

$$
\begin{aligned}
\left(\frac{d S}{d t}\right)_{a}= & N^{-1}<\frac{\int d \mathbf{r}^{N}}{V^{N}} e^{-\beta(W-A)} \sum_{i j} e^{i \mathbf{k} \cdot\left(\mathbf{r}_{i}-\mathbf{r}_{j}\right)} \\
& \times\left\{-\mathbf{k} \cdot \sum_{l}\left(\mu_{j l} \cdot \mathbf{F}_{l}\right)-\mathbf{k} \cdot\left[\mathrm{D}_{0}+k_{B} T \mu_{i j}\right] \cdot \mathbf{k}\right. \\
& -\mathbf{k} \cdot \Delta \mathrm{D} \cdot \mathbf{k}\} .
\end{aligned}
$$

Here the sum on $l$ includes $l=i$, the mobilities $\mu$ may depend on the coordinates of more than two particles, and in the end only terms of order $k^{2}$ will be retained. As before, ${ }^{19}$ the forces can be eliminated by the identity

$$
e^{-\beta(W-A)} \mathbf{F}_{l}=k_{B} T \nabla_{l}\left[e^{-\beta(W-A)}\right],
$$

and an integration by parts, yielding

$$
\begin{aligned}
& \left(\frac{d S}{d t}\right)_{a}=N^{-1} k_{B} T \int \frac{d r^{N}}{V^{N}} e^{-\beta(W-A)} \\
& \left.\times\left\{\sum_{i, j, l} i \mathbf{k} \nabla_{l}:\left[e^{i \mathbf{k} \cdot \mathbf{r}_{i j}} \mu_{j l}\right]\right\}-\sum_{i, j} e^{i \mathbf{k} \cdot \mathbf{r}_{i j}} \mathbf{k} \cdot \mu_{j j} \cdot \mathbf{k}\right\}-\mathbf{k} \cdot \Delta \mathrm{D} \cdot \mathbf{k}, \\
& \text { where } \mathbf{r}_{i j}=\mathbf{r}_{i}-\mathbf{r}_{j} .
\end{aligned}
$$

Equation (A8) may be simplified. In particular, the $l=j \neq i$ terms of the first sum, with $\nabla_{l}$ acting on the exponential, cancel the terms of the second sum with $i \neq j$. Terms of the first sum with $l=i \neq j$, and $\nabla_{l}$ acting on the exponential, may be combined with the surviving terms of the second sum. From Eqs. (A4a) and (A5), 


$$
\begin{aligned}
& \left(\frac{d S}{d t}\right)_{a}=N^{-1}\left(\sum_{i j} e^{i \mathbf{k} \cdot \mathbf{r}_{i j}}\left(-\mathbf{k} \cdot \mathrm{D}_{i j} \cdot \mathbf{k}\right)\right. \\
& \left.+\sum e^{i \mathbf{k} \cdot \mathbf{r}_{i j}} \mathbf{k} \mathbf{k} \nabla_{l}:\left[\mathrm{D}_{j l}\right]\right\rangle-\mathbf{k} \cdot \Delta \mathrm{D} \cdot \mathbf{k} .
\end{aligned}
$$

As stressed by Felderhof, $\nabla_{l} \cdot D_{j l} \neq 0$ if one goes to higher order in $(a / r)$.

Equation (25b) results from the substitution of Eq. (23b) into Eq. (20). This equation differs from Eq. (3) by the absence of the term of Eq. (3) in $\mathbf{S}_{j}$. This difference is not physically significant. Equations (23b) and (25b) contain the true diffusion tensor appropriate to moving particles in a closed volume, while in Eq. (3) Beenacker and Mazur ${ }^{5}$ were obliged to use the diffusion tensor appropriate to an infinite volume of fluid. The $-\Sigma_{j} S_{j}$ corrects for the difference in boundary conditions.

'M. M. Kops-Werkhofen and H. M. Fijnaut, J. Chem. Phys. 74, 1618 (1981).

${ }^{2}$ M. M. Kops-Werkhofen, H. J. Mos, P. N. Pusey, and H. M. Fijnaut,
Chem. Phys. Lett. 81, 365 (1981).

${ }^{3}$ M. M. Kops-Werkhofen and H. M. Fijnaut, J. Chem. Phys. 71, 2242 (1982).

${ }^{4} \mathrm{P}$. Mazur and W. van Saarlos, Physica A 115, 21 (1982).

${ }^{5}$ C. W. J. Beenacker and P. Mazur, Phys. Lett. A 91, 290 (1982).

${ }^{6} \mathrm{Z}$. Akcasu and H. Gurol, J. Polym. Sci. Polym. Phys. Ed. 14, 1 (1976).

${ }^{7}$ B. J. Ackerson, P. N. Pusey, and R. J. A. Tough, J. Chem. Phys. 76, 1279 (1982).

${ }^{8}$ G. D. J. Phillies, J. Chem. Phys. 74, 2436 (1981).

${ }^{9}$ S. Chandrasekhar, Rev. Mod. Phys. 15, 1 (1943).

${ }^{10}$ S. A. Rice and P. Gray, The Statistical Mechanics of Simple Liquids (Wiley, New York, 1965).

"'B. U. Felderhof, Physica A 89, 373 (1977).

${ }^{12} \mathrm{C}$. W. J. Beenacker and P. Mazur, Physica A 120, 388 (1983).

${ }^{13}$ G. D. J. Phillies, J. Chem. Phys. 79, 2325 (1983).

${ }^{14}$ P. N. Pusey and R. J. A. Tough, J. Phys. A 15, $1291\{1982\}$.

${ }^{15}$ S. Chandrasekhar, Rev. Mod. Phys. 15 (1943), remarks following Eqs. (184) and (133).

${ }^{16}$ S. Chandrasekhar, Rev. Mod. Phys. 15 (1943), point (i) following Eq. (133); Eq. (247).

${ }^{17}$ S. Chandrasekhar, Rev. Mod. Phys. 15 (1943), Eqs. (240)-(249) and (303)(316).

${ }^{18}$ B. J. Ackerson, J. Chem. Phys. 69, 684 (1978).

${ }^{19}$ G. D. J. Phillies, J. Chem. Phys. 77, 2623 (1982). 